\title{
Levomepromazine for Schizophrenia
}

\author{
Parthipan Sivaraman ${ }^{1, *}$, Ranganath Rattehalli ${ }^{2}$, and Mahesh Jayaram ${ }^{2}$ \\ ${ }^{1}$ South East Continuing Needs Service, Sheffield Health and Social Care NHS Foundation Trust, UK; ${ }^{2}$ General Adult Psychiatry, Leeds \\ Partnerships NHS Foundation Trust, UK.
}

*To whom correspondence should be addressed; tel: +44(0)1143050713, fax: +44(0)1142716450, e-mail: doctor.parthi@gmail.com

Key words: systematic review/meta-analysis/randomized clinical trials

\section{Background}

Levomepromazine is an "older" typical antipsychotic medication licensed for use in schizophrenia but sparingly prescribed in the United Kingdom.

\section{Objectives}

To determine the clinical effects and safety of levomepromazine compared with placebo or antipsychotic medications for schizophrenia and schizophreniform psychoses.

\section{Search Strategy}

We searched the Cochrane Schizophrenia Group Trials Register (December 2008), which is compiled by regular systematic searches of major databases including EMBASE, MEDLINE, and PsycINFO, the hand searching of relevant journals and conference proceedings, and searches of several key gray literature sources. References of all identified studies were inspected for further trials. We also contacted relevant pharmaceutical companies for additional information.

\section{Selection Criteria}

We included all the randomized clinical trials comparing levomepromazine with placebo or other antipsychotics for schizophrenia and schizophreniform psychoses.

\section{Data Collection and Analysis}

Data were extracted independently. For dichotomous outcomes, we calculated relative risk (RR) (randomeffects model), 95\% CI, and, where appropriate, number needed to treat (NNT) were calculated. We avoided the use of number needed to harm (NNH), as an alternative we used NNT for an additional beneficial outcome (NNTB) and number needed to treat for an additional harmful outcome (NNTH) to indicate the direction of effect. For continuous outcomes, we calculated weighted mean differences (WMD).

\section{Main Results}

The initial search resulted in 43 citations. We were able to include 10 of these that related to 4 randomized controlled trials (RCTs) with 192 participants in total. For our primary outcome of leaving the study early, levomepromazine was not significantly different compared with other antipsychotics. The levomepromazine arm was significantly better on Clinical Global Impression (CGI) severity compared with chlorpromazine $(n=38,1$ RCT, WMD: $-0.80 \mathrm{CI}:-1.51$ to -0.09 ) (figure 1). Risperidone was better for CGI endpoint scores $(n=42,1 \mathrm{RCT}$, RR: $2.33 \mathrm{CI}$ : 1.11-4.89, NNT: 3 CI: 2-10) compared with levomepromazine. Recipients given levomepromazine had a better Brief Psychiatric Rating Scale (BPRS) endpoint score ( $n=38,1$ RCT, WMD: -9.00 , CI: -17.46 to -0.54$)$ and Positive and Negative Syndrome Scale (PANSS) total score ( $n=38,1$ RCT, WMD: -15.90 , CI: -30.30 to -1.50$)$ than chlorpromazine. Risperidone recipients noticed a significant difference for the outcome "at least $20 \%$ reduction"

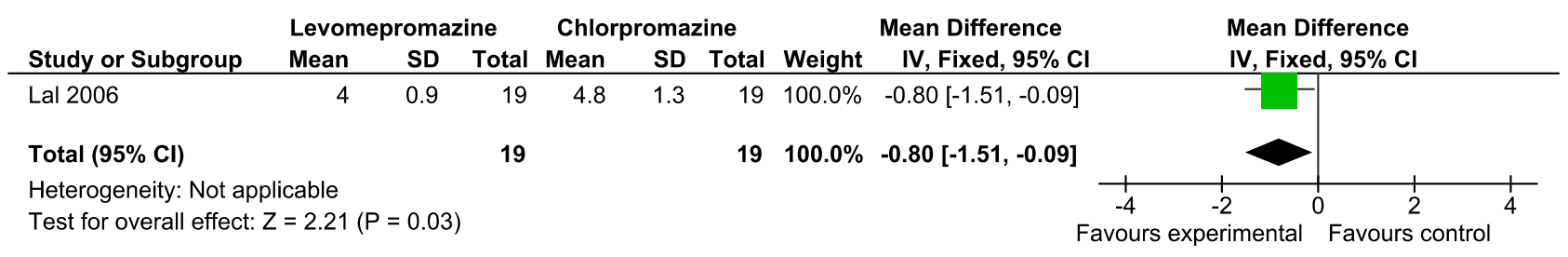

Fig. 1. Global effect: CGI severity (high = poor)-long term. 


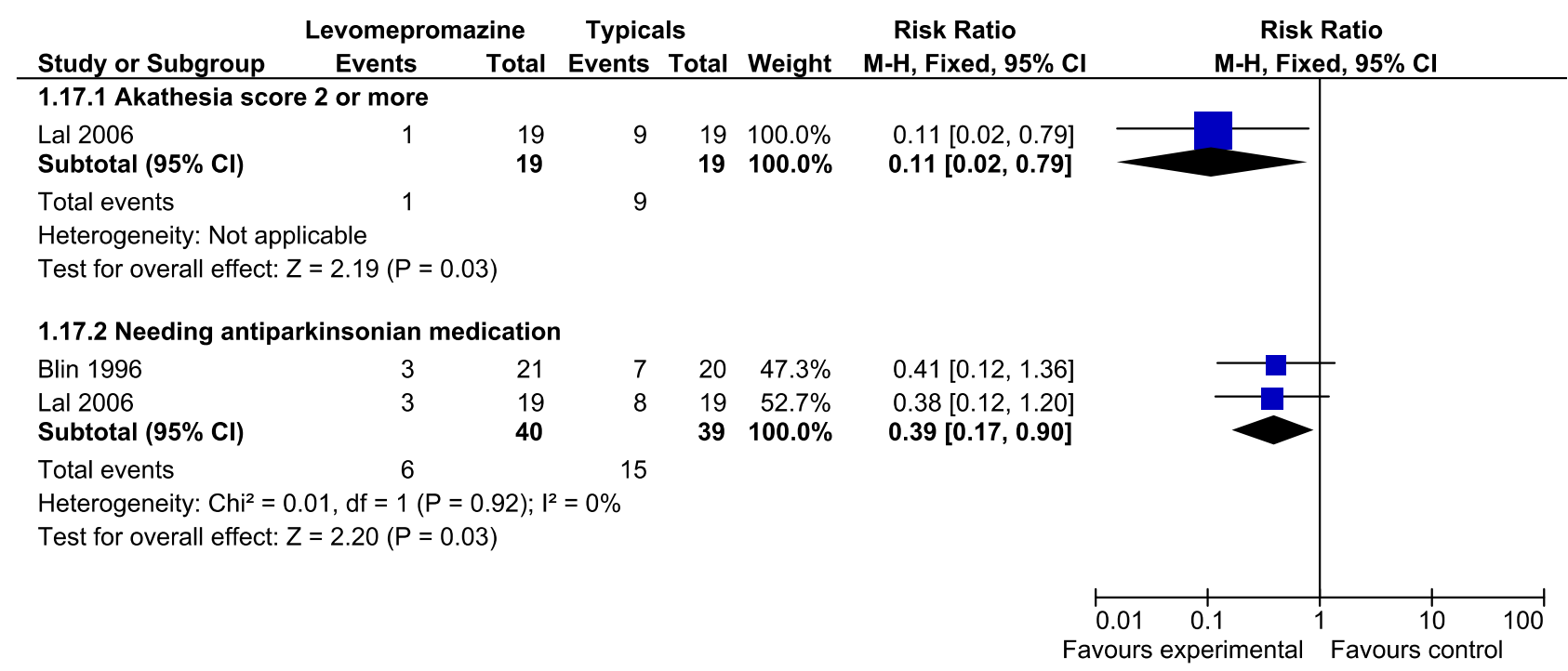

Fig. 2. Adverse events: extrapyramidal side effects.

on BPRS endpoint score ( $n=42,1$ RCT, RR: 3.33 CI: $1.07-$ 10.42, NNT: 3 CI: $2-14$ ) compared with levomepromazine. With regards to extrapyramidal side effects, levomepromazine had a significant advantage over typical antipsychotics $(n=79,2$ RCTs, RR: 0.39 CI: 0.17-0.90, NNTB 5 CI: $2-21)$ (figure 2). Levomepromazine caused less tremor $(n=41,1$ RCT RR: 0.12 CI: 0.02-0.87 NNTB 3 CI: 2-8), less antiparkinsonian medication administration $(n=79,2$ RCTs, RR: 0.39 CI: 0.17-0.90, NNTB 5, CI: 2-21) compared with haloperidol. Levomepromazine caused less akathisia compared with chlorpromazine but more hypotension compared with risperidone $(n=42,1 \mathrm{RCT}$, RR: $2.50 \mathrm{CI}$ : 1.21-5.18, NNTH 3, CI: 2-7). Dizziness was common with levomepromazine compared with other antipsychotic medications. Full version of this review is published on the Cochrane Library. ${ }^{1}$

\section{Authors' Conclusion}

Most data concerning levomepromazine in this review relates to short treatment period, and the data are too little to draw any conclusion about the effectiveness of levomepromazine when compared with other antipsy- chotics. Side effects such as the possibility of agranulocytosis and metabolic side effects still need to be studied thoroughly. There is no data comparing levomepromazine with placebo. Levomepromazine is comparable to chlorpromazine in its efficacy and caused less extrapyramidal side effects compared with haloperidol and chlorpromazine, clinicians could consider it as another drug available for the treatment of schizophrenia. As this review only had one trial in each of the comparisons, we are unable to make any further recommendations at this stage.

\section{Acknowledgment}

The authors have declared that there are no conflicts of interest in relation to the subject of this study.

\section{Reference}

1. Sivaraman P, Rattehalli RD, Jayaram MB. Levomepromazine for schizophrenia. Cochrane Database Syst Rev. 2010;(10): Art No.: CD007779. doi: 10.1002/14651858.CD007779.pub2. 